\title{
Traitements pharmacologiques et non pharmacologiques de la douleur neuropathique : une synthèse des recommandations françaises
}

\author{
Pharmacological and Non-Pharmacological Treatment for Neuropathic Pain: Short Form French \\ Guidelines
}

\author{
X. Moisset $\cdot$ D. Bouhassira $\cdot$ J. Avez Couturier $\cdot$ H. Alchaar $\cdot$ S. Conradi $\cdot$ M.-H. Delmotte $\cdot$ M. Lantéri-Minet \\ - J.-P. Lefaucheur - G. Mick · V. Piano - G. Pickering · E. Piquet - C. Regis · E. Salvat · N. Attal \\ (C) Lavoisier SAS 2020
}

Résumé Les douleurs neuropathiques (DN) restent très difficiles à soulager. Plusieurs recommandations ont été proposées au cours de ces dernières années, mais aucune n'a pris en compte à ce jour l'ensemble des approches thérapeutiques

X. Moisset $(\bowtie) \cdot$ M. Lantéri-Minet $\cdot$ G. Pickering

Inserm, Neuro-Dol, université Clermont-Auvergne,

58, rue Montalembert, F-63000 Clermont-Ferrand, France

e-mail : xavier.moisset@gmail.com

\section{Moisset}

CHU de Clermont-Ferrand,

F-63000 Clermont-Ferrand, France

D. Bouhassira $\cdot$ N. Attal

Inserm U987 et CETD, hôpital Ambroise-Paré,

F-92100 Boulogne-Billancourt, France

Université Versailles-Saint-Quentin-en-Yvelines,

F-78000 Versailles, France

J. Avez Couturier

Service de neuropédiatrie, consultation douleur enfant,

CIC-IT 1403, CHRU de Lille, F-59000 Lille, France

H. Alchaar

73, boulevard de Cimiez, F-06000 Nice, France

S. Conradi

CETD, CHRU de Nancy, F-54035 Vandœuvre-lès-Nancy, France

M.-H. Delmotte

GHU Paris, site Sainte-Anne, structure douleurs,

1, rue Cabanis, F-75014 Paris, France

M. Lantéri-Minet $\cdot$ E. Piquet

Département d'évaluation et traitement de la douleur, centre hospitalier universitaire (CHU) de Nice, Fédération hospitalo-universitaire InovPain, université Côte d'Azur, F-06000 Nice, France disponibles. Nous avons réalisé une revue systématique portant sur toutes les études concernant le traitement des DN périphériques et centrales de l'adulte et de l'enfant, publiées jusqu'en janvier 2018 et avons évalué la qualité des études et

J.-P. Lefaucheur
EA 4391, faculté de médecine, université Paris Est-Créteil,
F-94000 Créteil, France

Service de physiologie, explorations fonctionnelles, hôpital Henri-Mondor,

Assistance publique-Hôpitaux de Paris, F-94010 Créteil, France

G. Mick

Centre d'évaluation et traitement de la douleur du Voironnais, CHU de Grenoble-Alpes, F-38506 France

Laboratoire P2S, université Lyon-I, F-69100 Lyon, France

V. Piano

Service d'algologie, centre hospitalier de Draguignan, route de Montferrat, F-83007 Draguignan cedex, France

G. Pickering

Service de pharmacologie clinique, CPC/CIC Inserm 1405, $\mathrm{CHU}$ de Clermont-Ferrand, F-63000 Clermont-Ferrand, France

C. Regis

Centre d'évaluation et de traitement de la douleur,

CHU de Montpellier, F-34295 Montpellier, France

E. Salvat

Centre d'évaluation et de traitement de la douleur,

hôpitaux universitaires de Strasbourg, université de Strasbourg, Institut des neurosciences cellulaires et intégratives,

Centre national de la recherche scientifique, F-67000 Strasbourg, France 
le niveau de preuve des traitements au moyen du système GRADE. Les principaux critères d'inclusion étaient l'existence d'une DN chronique ( $\geq 3$ mois), une méthodologie contrôlée et randomisée, un suivi supérieur ou égal à trois semaines, un nombre de patients supérieur ou égal à dix par groupe et une évaluation en double insu pour les traitements pharmacologiques. Sur la base du GRADE, nous recommandons en première intention les antidépresseurs inhibiteurs mixtes de recapture des monoamines (duloxétine et venlafaxine), les antidépresseurs tricycliques, la gabapentine pour toute DN, et les emplâtres de lidocaïne $5 \%$ et la stimulation électrique transcutanée pour les DN périphériques localisées. Nous recommandons en deuxième intention la prégabaline, le tramadol (avec les précautions d'emploi afférentes aux opioïdes) et certaines associations pharmacologiques (antidépresseurs et gabapentine ou prégabaline) pour toute $\mathrm{DN}$, et les patchs de haute concentration de capsaïcine ( $8 \%$ ) et la toxine botulique de type A (en milieu spécialisé) pour les DN périphériques localisées. Nous recommandons en troisième intention la stimulation magnétique transcrânienne répétitive à haute fréquence du cortex moteur (en milieu spécialisé) et les opioïdes forts (en l'absence d'alternative et en respectant les précautions d'emploi afférentes aux opioïdes) pour toute douleur neuropathique, et la stimulation médullaire pour les douleurs radiculaires chroniques postchirurgicales et la polyneuropathie diabétique douloureuse. La psychothérapie (thérapie cognitivocomportementale et thérapie de pleine conscience) peut être recommandée en deuxième intention en association avec les traitements précédents.

Mots clés Douleur neuropathique . Recommandations . Pharmacothérapie . Psychothérapie . Neurostimulation

\begin{abstract}
Neuropathic pain remains a significant unmet medical need. Several recommendations have recently been proposed concerning pharmacotherapy, neurostimulation techniques and interventional management, but no comprehensive guideline encompassing all these treatments has yet been issued. We have performed a systematic review of pharmacotherapy, neurostimulation, surgery, psychotherapies and other types of therapy for peripheral or central neuropathic pain, based on studies published in peer-reviewed journals before January 2018. The main inclusion criteria were chronic neuropathic pain for at least three months, a randomized controlled methodology, at least three weeks of follow-up with at least 10 patients per group, and a doubleblind design for drug therapy. Based on the GRADE system, we provide weak-to-strong recommendations for use and proposal as a first-line treatment for SNRIs (duloxetine and venlafaxine), gabapentin and tricyclic antidepressants, and for topical lidocaine and transcutaneous electrical nerve stimulation specifically for peripheral neuropathic pain; a weak
\end{abstract}

recommendation for use and proposal as a second-line treatment for pregabalin, tramadol, combination therapy (antidepressant combined with gabapentinoids), and for highconcentration capsaicin patches and botulinum toxin A specifically for peripheral neuropathic pain; a weak recommendation for use and proposal as a third-line treatment for highfrequency rTMS of the motor cortex, spinal cord stimulation (failed back surgery syndrome and painful diabetic polyneuropathy) and strong opioids (in the absence of an alternative). Psychotherapy (cognitive behavioral therapy and mindfulness) is recommended as a second-line therapy, as an add-on to other therapies. An algorithm encompassing all the recommended treatments has been proposed.

Keywords Neuropathic pain . Recommendations . Pharmacotherapy . Psychotherapy . Neurostimulation

\section{Introduction}

La douleur neuropathique (DN) est secondaire à une lésion ou à une maladie affectant le système nerveux somatosensoriel. Elle a un impact considérable sur la qualité de vie et est à l'origine d'un coût socio-économique majeur [1-4]. Cependant, de nombreux patients présentant une DN ne reçoivent pas de traitement pharmacologique approprié [2,5-9]. De nombreuses raisons peuvent être avancées, notamment une méconnaissance du diagnostic de $\mathrm{DN}$ ou un manque de connaissance des molécules efficaces et de leurs modalités d'utilisation. Par ailleurs, les approches non pharmacologiques telles que la neurostimulation, la psychothérapie et les techniques dites complémentaires (hypnose, acupuncture...) sont de plus en plus souvent proposées aux patients douloureux neuropathiques, simultanément ou successivement $[10,11]$. Cependant, les recommandations pour la prise en charge de la DN ont toujours été limitées à un type de traitement spécifique, notamment les traitements pharmacologiques ou la neurostimulation. Ainsi, les dernières recommandations françaises pour le traitement de la douleur neuropathique ont ciblé les approches pharmacologiques [12]. Depuis ces recommandations publiées en 2010, des recommandations internationales fondées sur les preuves ont été diffusées pour les traitements pharmacologiques [13], les techniques interventionnelles [14] et la neurostimulation centrale [15]. Aucune recommandation prenant en compte l'ensemble des techniques disponibles pour la prise en charge de la DN n'a été élaborée jusqu'à présent. De plus, la majorité des recommandations précédentes n'ont pris en compte que les études réalisées chez l'adulte, et pas les patients pédiatriques ou très âgés. En France, le traitement de la douleur neuropathique en pédiatrie est fondé sur les recommandations de l'AFFSSAPS de 2009 [16], qui sont 
principalement issues de recommandations faites chez l'adulte et d'avis d'experts.

Le présent document correspond à une version synthétique des nouvelles recommandations françaises publiées en langue anglaise en 2020, qui intègrent l'ensemble des options thérapeutiques disponibles pour la DN [17].

\section{Méthodes}

Le détail des méthodes est disponible dans l'article complet [17]. En résumé, le système GRADE (Grading of Recommendations Assessment, Development, and Evaluation) $[18,19]$ a été utilisé pour aboutir à un algorithme thérapeutique global tenant compte de tous les traitements pharmacologiques, psychothérapeutiques ou interventionnels pour la prise en charge de la DN. L'élaboration de ces recommandations a été soutenue par plusieurs sociétés savantes (Société française d'étude et de traitement de la douleur, SFETD ; Société française de neurologie, SFN ; Association nationale pédagogique des enseignants en thérapeutique, APNET).

Nous avons réalisé une revue systématique de tous les essais randomisés contrôlés (placebo ou comparatifs), réalisés en double insu comportant au moins trois semaines de suivi. La recherche a été réalisée le 18 janvier 2018 dans PubMed/MEDLINE et Embase. Une recherche complémentaire a été réalisée dans les mêmes bases de données en août 2019. Les études en simple insu et les études ouvertes n'ont été considérées que pour les traitements pour lesquels une méthodologie en double insu était difficile ou impossible (psychothérapie, chirurgie). Pour les traitements pharmacologiques topiques ou oraux, la recherche a débuté en juin 2013, les données antérieures à 2013 étant fondées sur la dernière revue systématique avec méta-analyse publiée en 2015, réalisée avec la même méthodologie et codirigée par un membre de notre groupe de travail [13]. Pour les autres traitements, les articles publiés depuis 1966 (premières études publiées dans MEDLINE) ont été évalués. Les traitements à visée étiopathogénique (par exemple traitements de la neuropathie diabétique) de même que les traitements de la névralgie essentielle du trijumeau [20] ou de l'érythromélalgie (qui ne fait pas partie stricto sensu des douleurs neuropathiques) ont été exclus de ce travail.

L'ensemble du texte a été validé par les 15 membres du groupe de travail. Une première relecture avec cotation a été effectuée par les 33 membres du groupe de lecture. À la suite de ce premier retour, des modifications ont été effectuées, et un deuxième tour de cotation a été réalisé. Toutes les propositions ont été jugées appropriées par le groupe de lecture (score médian $\geq 7 / 9$ ). Un accord relatif (scores entre 5 et 9) ou fort (scores entre 7 et 9 ) pour au moins $90 \%$ des membres du groupe de lecture a été obtenu pour toutes les propositions sauf pour la carbamazépine. Les détails sont disponibles dans la publication originale.

\section{Résultats}

\section{Études sélectionnées}

Un total de 131 articles a été inclus, dont $47 \%$ portaient sur la polyneuropathie diabétique ou la douleur postzostérienne, $11 \%$ sur la douleur centrale et $42 \%$ sur d'autres types de douleur (traumatique, radiculaire, liée au VIH, postchimiothérapie, étiologies multiples). Nous présentons ici une synthèse des résultats dont les détails sont disponibles dans la publication originale.

\section{Traitements pharmacologiques oraux et topiques}

\section{Antidépresseurs}

Les inhibiteurs de recapture de la sérotonine et de la noradrénaline (IRSNA), duloxétine et venlafaxine, et les antidépresseurs tricycliques ont un niveau élevé de recommandation dans les DN périphériques et centrales. L'utilisation de la duloxétine est préférable à celle de la venlafaxine en raison du plus grand nombre d'études positives de bonne qualité. Les tricycliques doivent être monitorés lorsqu'ils sont utilisés à fortes doses $(>75 \mathrm{mg} / \mathrm{j})$ en raison de leurs risques potentiels d'hypotension orthostatique et de problèmes cardiaques (troubles de la conduction, décompensation d'un infarctus du myocarde récent) et ne sont pas recommandés à ces doses chez les patients de plus de 70 ans. L'utilisation de doses faibles à modérées $(10-50 \mathrm{mg} / \mathrm{j})$ est donc préférable, car ils peuvent être efficaces à ces doses.

\section{Antiépileptiques}

La gabapentine a un niveau élevé de recommandation dans les DN périphériques et à moindre degré centrales. Il existe une recommandation faible pour l'utilisation de la prégabaline, la majorité des études de bonne qualité réalisées récemment étant négatives sur le critère primaire, avec en outre des effets indésirables plus fréquents que pour la gabapentine selon une étude comparative. Il est impossible de conclure concernant l'efficacité des autres antiépileptiques, même si l'oxcarbazépine ou le lacosamide semblent avoir une efficacité dans des sous-groupes de patients. Nos critères d'inclusion n'ont pas permis de retenir d'études concernant le clonazépam, ce qui n'exclut pas une efficacité potentielle de ce traitement sur les paroxysmes douloureux (études ouvertes). 


\section{Opiö̈des}

Les opioïdes forts (en particulier la morphine et l'oxycodone) et le tramadol ont un niveau de recommandation faible, alors que les données concernant la buprénorphine et le fentanyl ne sont pas concluantes. Le tapentadol (non disponible en France à l'heure actuelle) a un niveau de recommandation faible en faveur de son utilisation dans les $\mathrm{DN}$ périphériques.

\section{Traitements topiques}

Les emplâtres de lidocaïne $5 \%$ et les patchs de haute concentration de capsaïcine ( $8 \%$ ) ont un niveau de recommandation faible dans les DN périphériques. Il n'est pas possible de conclure concernant l'efficacité d'autres traitements topiques (en particulier capsaïcine faible concentration, nitroglycérine, clonidine et kétamine topique).

\section{Toxine botulique de type A}

La toxine botulique de type A en injections sous-cutanées a un niveau de recommandation faible, malgré deux études positives récentes de qualité élevée, en l'absence de larges études multicentriques.

\section{Associations thérapeutiques}

Il existe une recommandation faible en faveur de l'utilisation de l'association d'antidépresseurs (IRSNA ou tricycliques) avec des gabapentinoïdes (gabapentine ou prégabaline) ou de la morphine ainsi que de la combinaison de gabapentinoïdes et d'opioïdes. L'efficacité d'autres associations n'est pas établie formellement.

\section{Cannabinoüdes}

Il est impossible de conclure concernant l'efficacité des cannabinoïdes (nabiximols transmuqueux, tétrahydrocannabinol oral) du fait d'un nombre important d'études négatives de bonne qualité. Cela n'exclut pas leur efficacité sur des sous-groupes de patients ou des symptômes particuliers tels que les douleurs paroxystiques.

\section{Manque de données}

Nous n'avons retrouvé aucune étude satisfaisant à nos critères d'inclusion concernant l'utilisation du néfopam, du paracétamol, des anti-inflammatoires non stéroïdiens (AINS) et du midazolam dans les DN.
Kétamine orale, traitements intraveineux, blocs nerveux et traitements par voie intrathécale

\section{Kétamine orale}

Il est impossible de conclure quant à l'efficacité de la kétamine par voie orale du fait d'un faible nombre d'études, de qualité médiocre.

\section{Traitements intraveineux par lidocaïne ou kétamine}

Compte tenu de nos critères d'inclusion stricts comportant notamment un suivi d'efficacité jusqu'à trois semaines, il est impossible de conclure quant à l'efficacité de la kétamine et de la lidocaïne par voie intraveineuse. Cependant, certaines études contrôlées ont rapporté une efficacité à court terme de ces traitements (jusqu'à deux semaines après une administration unique de ces molécules), suggérant leur intérêt pour la prise en charge des patients en crise ou présentant une exacerbation récente de leur douleur.

\section{Blocs nerveux}

Compte tenu d'un nombre insuffisant d'études en double insu avec un suivi suffisant, il est impossible de conclure concernant l'efficacité des blocs nerveux sur les DN.

\section{Traitements par voie intrathécale}

Une étude japonaise a suggéré l'efficacité de la méthylprednisolone par voie intrathécale dans les douleurs postzostériennes, mais elle a été controversée et n'a jamais été répliquée, ce qui ne permet pas de conclure. Nous n'avons retrouvé aucune étude satisfaisant à nos critères d'inclusion concernant la ziconotide, la morphine et la clonidine dans les douleurs neuropathiques, car la totalité des études en double insu utilisant ces traitements ont porté sur la douleur chronique réfractaire en général et non spécifiquement sur les DN.

Techniques de neurostimulation et procédures invasives

\section{Neurostimulation périphérique non invasive}

L'utilisation de la stimulation électrique transcutanée (TENS pour transcutaneous electrical nerve stimulation) a un niveau de recommandation faible en faveur de son utilisation dans les DN périphériques. En revanche, l'utilisation des champs magnétiques statiques ou pulsés a un niveau de recommandation faible contre leur utilisation. 


\section{Neurostimulation centrale non invasive}

Il existe une recommandation faible en faveur de l'utilisation de la stimulation magnétique transcrânienne répétitive à haute fréquence (rTMS pour repetitive transcranial magnetic stimulation) du cortex moteur primaire. Il n'est pas possible de conclure concernant l'efficacité de la rTMS du cortex dorsolatéral préfrontal, de la tDCS (transcranial direct current stimulation), de la tsDCS (transcutaneous spinal direct current stimulation) et de la CES (cranial electrotherapy stimulation). Il existe une recommandation faible contre l'utilisation de la rTMS de l'insula postérieure ou du cortex cingulaire antérieur.

\section{Radiofréquence pulsée}

Il existe une recommandation faible en faveur de l'utilisation de la radiofréquence pulsée (PRF) dans les douleurs postzostériennes thoraciques. Il n'est pas possible de conclure concernant l'utilisation de la PRF pour les douleurs radiculaires.

\section{Neurostimulation invasive périphérique}

Il n'est pas possible de conclure à ce jour concernant l'efficacité de la neurostimulation des nerfs périphériques ou de la stimulation du ganglion sensitif dorsal (dorsal root ganglion, DRG) dans les DN.

\section{Neurostimulation centrale invasive}

Toutes les études randomisées contrôlées (avec groupe témoin) portant sur la stimulation médullaire (SCS, spinal cord stimulation) dans les douleurs radiculaires chroniques postchirurgicales (FBSS, failed back surgery syndrome) et la douleur neuropathique liée au diabète étaient positives, avec des tailles d'effets importants et un faible risque d'effet indésirable. Cependant, ces études étaient toutes ouvertes (dans la mesure où l'aveugle complet est impossible avec cette technique de stimulation qui induit des paresthésies) et ont généralement comparé la SCS à la prise en charge en soins courants. Ainsi, la SCS apparait efficace dans ces douleurs mais avec un niveau de preuve faible. Il n'est pas possible de conclure concernant l'efficacité de la stimulation invasive du cortex moteur dans la mesure où seules les études ouvertes sont positives.

\section{Autres techniques chirurgicales}

Il n'est pas possible de conclure quant à l'efficacité de la libération d'adhérences épidurales, de la diathermie à ondes longues et de la décompression nerveuse. Aucune étude satisfaisant à nos critères d'inclusion n'a concerné l'utilisa- tion de la DREZotomie (dorsal root entry zone), technique chirurgicale de thermocoagulation sélective des racines à leur entrée dans la corne postérieure de la moelle. Cela n'exclut pas son intérêt potentiel pour les douleurs de type avulsion du plexus brachial et lésion de la queue de cheval (études de cohortes observationnelles ouvertes positives).

\section{Psychothérapie}

Six études ont été sélectionnées, toutes de qualité modérée, apportant aux patients un bénéfice, celui-ci pouvant perdurer au-delà de l'intervention [21]. Le cadre psychothérapeutique était différent d'une étude à une autre, et plusieurs ont été réalisées en groupe. Il existe donc un niveau de recommandation faible en faveur de l'utilisation de la thérapie cognitivocomportementale (TCC) et de la thérapie de pleine conscience, en complément des traitements précédents efficaces.

\section{Autres traitements non pharmacologiques}

Aucune conclusion n'est possible concernant l'utilisation de l'aromathérapie, de l'auriculothérapie et du laser à faible énergie, du fait de données émanant d'études uniques de qualité faible ou modérée. Il existe une recommandation faible contre l'utilisation de la vitamine E. Aucune étude satisfaisant à nos critères d'inclusion n'a concerné l'utilisation d'autres techniques complémentaires. Cela n'exclut pas l'efficacité potentielle de la thérapie miroir, de l'hypnose et de l'acupuncture, ces techniques ayant fait la preuve de leur efficacité à court terme ( $<3$ semaines) ou dans des essais ne ciblant pas la DN.

\section{Études en population pédiatrique ou chez des sujets âgés}

Une seule étude comparative de petite taille a été identifiée en pédiatrie [22]. Celle-ci a montré que l'amitriptyline $10 \mathrm{mg} / \mathrm{j}$ ou la gabapentine $900 \mathrm{mg} / \mathrm{j}$ avaient la même efficacité sur la douleur neuropathique de l'enfant, sans générer d'effets indésirables majeurs. Une seule étude contrôlée randomisée portant spécifiquement sur la DN des personnes âgées a été identifiée : elle a montré un effet bénéfique de la thérapie de pleine conscience dans cette population par rapport à un groupe témoin bénéficiant d'une prise en charge en soins courants.

\section{Algorithme thérapeutique chez l'adulte}

Sur la base de notre revue systématique, nous proposons un algorithme thérapeutique (Fig. 1) pour les traitements de première, deuxième et troisième intentions des douleurs neuropathiques périphériques ou centrales. Un tableau pour l'utilisation pratique de ces thérapeutiques est également proposé (Tableau 1). Les traitements recommandés ici ont tous un 
Tableau 1 Utilisation pratique des traitements pour la douleur neuropathique chronique en France

\section{A- Douleur neuropathique périphérique focale}

Première intention • Emplâtres de lidocaïne, 1 à 3 emplâtres selon l'étendue de la zone douloureuse, 12 heures par jour. Les effets indésirables principaux sont l'irritation cutanée ou l'allergie (AMM : douleurs neuropathiques postzostériennes chez l'adulte)

- Stimulation électrique transcutanée (TENS). En France, remboursement uniquement si la prescription est effectuée par une structure douleur chronique. Les effets indésirables principaux sont l'irritation cutanée ou l'allergie

Ces traitements peuvent être utilisés seuls ou en association avec des traitements systémiques (consensus d'experts) et sont à favoriser chez des patients avec une zone douloureuse peu étendue et une sensibilité résiduelle de leur aire douloureuse (c'est-à-dire sans anesthésie totale à la stimulation thermique ou mécanique dans la zone douloureuse)

Deuxième intention - Capsaïcine en patchs de haute concentration ( $8 \%$ ) [1-4 patchs selon l'extension de la zone douloureuse, appliqués 30 minutes sur les pieds et 60 minutes sur les autres zones du corps]. Les effets indésirables principaux incluent sensation de brûlure initiale (qui peut être réduite par l'application de froid), rougeur et œdème. De rares cas d'HTA ont été rapportés en lien avec la douleur provoquée par l'application. Ainsi, les patients doivent être surveillés pour la douleur et la tension artérielle pendant au moins une heure après l'application (AMM : douleurs neuropathiques périphériques chez l'adulte)

- Toxine botulique de type A (jusqu'à 300 unités selon l'extension de la zone douloureuse, en injections sous-cutanées). L'effet indésirable principal est lié à la douleur provoquée par les injections (AMM : dystonie cervicale, spasticité ; aucune AMM pour la douleur neuropathique)

Ces 2 traitements sont disponibles uniquement en usage hospitalier (hôpital de jour pour la capsaïcine). Ces traitements peuvent être utilisés seuls ou en association avec des traitements systémiques (consensus d'experts) et sont à favoriser chez des patients avec une zone douloureuse peu étendue et avec une sensibilité résiduelle (c'est-à-dire sans anesthésie totale à la stimulation thermique ou mécanique dans la zone douloureuse) ainsi qu'une allodynie mécanique pour la toxine botulique

\section{B- Toute douleur neuropathique, périphérique ou centrale, focale ou diffuse}

Première intention - Antidépresseurs IRSNA par voie orale : duloxétine (60-120 mg/j en 1 ou 2 prises) ou venlafaxine (150$225 \mathrm{mg} / \mathrm{j}$ en 2 ou 3 prises) avec une préférence pour la duloxétine (plus d'études de grande qualité positives). Les effets indésirables principaux incluent nausées, anorexie, constipation et troubles sexuels. De rares cas d'HTA ont été rapportés avec la venlafaxine à haute dose $(\geq 150 \mathrm{mg} / \mathrm{j})$. Une levée d'inhibition avec risque suicidaire est rare mais possible lors de l'initiation de tout traitement antidépresseur (AMM : douleurs neuropathiques diabétique périphérique pour la duloxétine, pas d'AMM pour la venlafaxine)

- Gabapentine orale (1 200-3 $600 \mathrm{mg} / \mathrm{j}$ en 3 prises). Les effets indésirables principaux incluent somnolence, vertiges, prise de poids et œdèmes périphériques. Les facteurs de risque de mésusage doivent être recherchés avant la prescription, et un mésusage ou un abus potentiel doivent être évalués à chaque renouvellement $\mathrm{du}$ fait du risque de mésusage avec ce traitement (AMM : douleurs neuropathiques périphériques telles que la neuropathie diabétique et la névralgie postzostérienne chez l'adulte)

- Antidépresseurs tricycliques par voie orale (amitriptyline, clomipramine, imipramine), $10-150 \mathrm{mg} / \mathrm{j}$ en 1 ou 2 prises. Des doses faibles $(<75 \mathrm{mg} / \mathrm{j})$ sont généralement efficaces. Les effets indésirables principaux incluent somnolence et prise de poids. Une levée d'inhibition avec risque suicidaire est rare mais possible lors de l'initiation de tout traitement antidépresseur. Ces traitements doivent être surveillés en raison de leur risque cardiaque, anticholinergique ou d'hypotension orthostatique, en particulier pour les patients présentant des comédications multiples ou des comorbidités et à des doses $>75 \mathrm{mg} / \mathrm{j}$. Chez les personnes âgées (> 70 ans), polymédiquées ou avec de multiples comorbidités, la posologie ne doit pas excéder $75 \mathrm{mg} / \mathrm{j}$ (AMM : douleurs neuropathiques périphériques de l'adulte pour l'amitriptyline : douleurs neuropathiques de l'adulte pour la clomipramine et l'imipramine)

Il est toujours important de débuter à faibles posologies et d'augmenter lentement les doses pour tous les traitements oraux afin de minimiser le risque d'effets indésirables. Les patients doivent être informés $\mathrm{du}$ risque de ces traitements pour la conduite automobile, en particulier lors de leur initiation 
Deuxième intention • Prégabaline orale (150-600 mg/j, en 2 ou 3 prises, comme alternative à la gabapentine en cas de problème de tolérance ou d'efficacité). Les effets indésirables principaux incluent somnolence, vertiges, prise de poids et œdèmes périphériques. Les facteurs de risque de mésusage doivent être recherchés avant la prescription, et un mésusage ou un abus potentiel doivent être évalués à chaque renouvellement du fait du risque de mésusage avec ce traitement (plus fréquents qu'avec la gabapentine) [AMM : douleurs neuropathiques périphériques et centrales chez l'adulte)

- Tramadol à libération prolongée par voie orale $(100-400 \mathrm{mg} / \mathrm{j}$ en 1 ou 2 prises par jour. Le tramadol a les mêmes effets indésirables et le même risque potentiel d'abus que les opiö̈des forts, bien que ces risques soient moins fréquents, la dose maximum de tramadol $(400 \mathrm{mg} / \mathrm{j})$ correspondant à 40 à $68 \mathrm{mg}$ en équivalent morphine (evidence.nhs.uk). Le tramadol peut être associé avec des antiépileptiques (consensus d'experts) mais doit être utilisé avec prudence en association à des antidépresseurs à forte posologie (risque potentiel de syndrome sérotoninergique) [AMM : douleurs modérées à sévères]

- Association d'antidépresseurs (tricycliques entre 25 et $75 \mathrm{mg} / \mathrm{j}$ ou duloxétine $60 \mathrm{mg} / \mathrm{j}$ )

et de gabapentinoïdes (prégabaline 150-300 mg/j ou gabapentine $1200-1800 \mathrm{mg} / \mathrm{j}$ )

- Thérapie cognitivocomportementale et thérapie pleine conscience. Parmi les psychothérapies, ces techniques ont le meilleur niveau de preuve et sont recommandées en association avec d'autres traitements (les essais contrôlés montrant l'efficacité de ces techniques ayant été réalisées chez des patients recevant des traitements pharmacologiques)

Il est toujours important de débuter à faibles posologies et d'augmenter lentement les doses pour tous les traitements oraux afin de minimiser le risque d'effets indésirables. Les patients doivent être informés du risque de ces traitements pour la conduite automobile, en particulier lors de leur initiation.

Troisième intention • rTMS à haute fréquence du cortex moteur $(80 \%$ du seuil moteur, $10 \mathrm{~Hz}, 1500$ à 3000 impulsions par séance). Peut être proposée pour une douleur localisée ou plus diffuse (par exemple lésion médullaire ou neuropathie diabétique). Cette technique est disponible uniquement dans des centres spécialisés. Le principal effet indésirable est le risque de céphalées dans les heures suivant la stimulation. Le risque de crise comitiale est minime

- La stimulation médullaire a été montrée efficace dans les syndromes de douleur chronique après chirurgie du rachis et les douleurs neuropathiques liées au diabète. Cette technique est disponible uniquement dans des centres spécialisés. Elle peut être envisagée avant l'utilisation d'opioïdes forts et après une année seulement d'évolution de la douleur. Comme toute technique invasive, l'utilisation de cette technique doit être précédée d'une évaluation psychologique du patient. Les complications sont rares et principalement liées à des problèmes matériels (par exemple migration, déconnexion ou casse d'électrode) pouvant nécessiter une chirurgie supplémentaire. Le risque de lésion médullaire, par lésion directe ou indirecte via un hématome épidural, est extrêmement faible

- Opioïdes forts (morphine et oxycodone à libération prolongée). Les opioïdes forts ne doivent être proposés qu'en l'absence d'alternatives. Ils nécessitent une évaluation minutieuse du risque potentiel d'abus, de préférence à l'aide d'un questionnaire dédié tel que l'opioid risk tool, et la prescription doit être la plus brève possible (quelques mois), avec une surveillance régulière. L'initiation doit être faite de préférence par un spécialiste de la douleur. L'augmentation de dose doit être progressive et ne doit pas excéder $150 \mathrm{mg}$ d'équivalent morphine par jour (consensus d'experts). Les opioïdes à libération immédiate doivent être évités, en particulier le fentanyl transmuqueux (sauf dans le cas de douleurs associées au cancer, dans le cadre de leur AMM), en raison d'un risque d'abus plus important. En l'absence d'amélioration significative concernant la douleur, la fonction ou la qualité de vie, les opioïdes doivent être réduits progressivement et arrêtés. Les effets indésirables principaux incluent constipation, sédation, nausées, prurit et dysurie. L'utilisation au long cours est associée avec le risque de mésusage, d'abus, d'hypogonadisme et d'hypocorticisme. Les facteurs de risque de mésusage doivent être recherchés avant l'initiation, tandis que l'abus et le mésusage doivent être évalués à chaque renouvellement. La prescription d'opioïdes doit être surveillée avec encore plus d'attention chez les hommes de moins de 50 ans, en raison des risques de mésusage et de risques gonadiques au long cours (AMM : douleurs persistantes intenses ou rebelles aux autres analgésiques, en particulier douleur d'origine cancéreuse pour morphine et oxycodone) - Combinaison d'opioïdes avec des antidépresseurs ou des gabapentinoïdes, en l'absence d'alternative. Les doses d'opioïdes (dont le tramadol) doivent être réduites en cas d'association avec des gabapentinoïdes en raison d'un surrisque de décès avec une telle association 


\section{C- Règles concernant le passage d'une ligne de traitement à une autre \\ Modification Aucune étude n'a défini les règles de passage à un traitement de deuxième ou de troisième intention. En thérapeutique l'absence de contre-indication, les traitements de première intention devraient être proposés en premier. En cas d'effets indésirables non tolérables ou de manque d'efficacité, définis comme une diminution de la douleur de moins de $30 \%$ et/ou une amélioration fonctionnelle de moins de $30 \%$ après un essai d'au moins 6 semaines à la dose maximale tolérée, un changement pour un traitement de deuxième intention peut être proposé (consensus d'experts). La même règle peut s'appliquer pour passer de la deuxième à la troisième intention (consensus d'experts).}

TENS : transcutaneous electrical nerve stimulation ; IRSNA : antidépresseur inhibiteur de recapture de la sérotonine et de la noradrénaline ; HTA : hypertension artérielle ; rTMS : repetitive transcranial magnetic stimulation ; AMM : autorisation de mise sur le marché

niveau de preuve faible ou élevé et sont disponibles en France. Les recommandations de cet algorithme tiennent également compte de leur facilité d'utilisation (ainsi, les traitements disponibles uniquement en milieu hospitalier à ce jour, comme les patchs de capsaïcine, sont recommandés en deuxième intention pour cette raison) et de l'ancienneté d'utilisation ou de l'expérience des traitements. En revanche, elles ne tiennent pas compte des autorisations de mise sur le marché (AMM) en France, dans la mesure où celles-ci ne sont pas nécessairement actualisées en fonction des nouvelles données d'efficacité clinique.

\section{Discussion}

Nous présentons ici une synthèse des recommandations françaises actualisées de 2020, soutenues par plusieurs sociétés savantes (SFETD, SFN, APNET), dont la version complète est disponible en anglais [17]. L'utilisation du système GRADE nous a permis de combiner des études de qualité hétérogène et de présenter le premier algorithme tenant compte de toutes les approches thérapeutiques possibles, ce qui est une nouveauté et une force indéniable de ce travail.

Les précédentes recommandations proposées par la SFETD en 2010 [12] étaient surtout focalisées sur la pharmacothérapie. Ces recommandations proposaient les antidépresseurs tricycliques, la gabapentine et la prégabaline en première intention pour toute $\mathrm{DN}$, les patchs de lidocaïne en première intention pour les $\mathrm{DN}$ périphériques localisées (notamment la douleur postzostérienne) et la duloxétine spécifiquement pour les neuropathies périphériques diabétiques douloureuses. Le tramadol était proposé en deuxième intention et les opioïdes forts en troisième intention.

Comme dans les recommandations précédentes, nous proposons la gabapentine et les antidépresseurs tricycliques en première intention, malgré un profil d'effets indésirables médiocre pour les tricycliques, du fait de leur efficacité possible à faibles doses et de très nombreuses preuves d'efficacité. Nous recommandons plus largement désormais l'usage des antidépresseurs IRSNA, notamment la duloxétine dans les douleurs neuropathiques périphériques ou centrales, du fait de plusieurs études récentes positives de bonne qualité, alors que l'AMM française de ce traitement est limitée aux douleurs neuropathiques du diabétique. Enfin, nous recommandons les emplâtres de lidocaïne en première intention pour les DN périphériques localisées, même si ce traitement a une AMM limitée en France aux DN postzostériennes, du fait de deux études récentes positives dans des douleurs neuropathiques périphériques d'étiologies variées, notamment en cas d'allodynie [23,24].

En revanche, nos nouvelles recommandations diffèrent à plusieurs titres des recommandations de 2010. L'évolution la plus notable concerne la place de la prégabaline, qui est désormais proposée en troisième intention, et ce pour plusieurs raisons. D'une part, la majorité (11 sur 14) des études contrôlées de la prégabaline depuis cinq ans sont négatives, et les études positives ont une taille d'effet très faible [25]. En outre, l'efficacité et le profil d'effets indésirables semblent bien moins favorables pour la prégabaline par rapport à la gabapentine selon une étude comparative en double insu réalisée dans la sciatique chronique [26]. Enfin, de nombreuses études récentes depuis ces cinq dernières années ont fait état d'un risque d'usage détourné et de conduites addictives, en lien avec l'utilisation de gabapentinoïdes, qui semble plus important avec la prégabaline [27]. Cependant, un risque de mésusage existe aussi avec la gabapentine, et nous recommandons désormais d'évaluer le risque d'abus et de mésusage pour toute prescription de gabapentinoïdes, comme c'est le cas pour les opioïdes.

La deuxième évolution notable est liée à l'émergence de deux nouveaux traitements pharmacologiques utilisables par voie topique ou locale depuis ces dernières années : les patchs de haute concentration de capsaïcine et la toxine botulique A. Les patchs de haute concentration de capsaïcine, commercialisés en France depuis 2011, ont fait l'objet de plusieurs études positives dans les douleurs neuropathiques périphériques et disposent d'une AMM dans cette indication. La toxine botulique A, dont l'efficacité est largement établie dans le traitement de la spasticité ou de la dystonie, a fait l'objet de plusieurs études positives récentes indiquant 


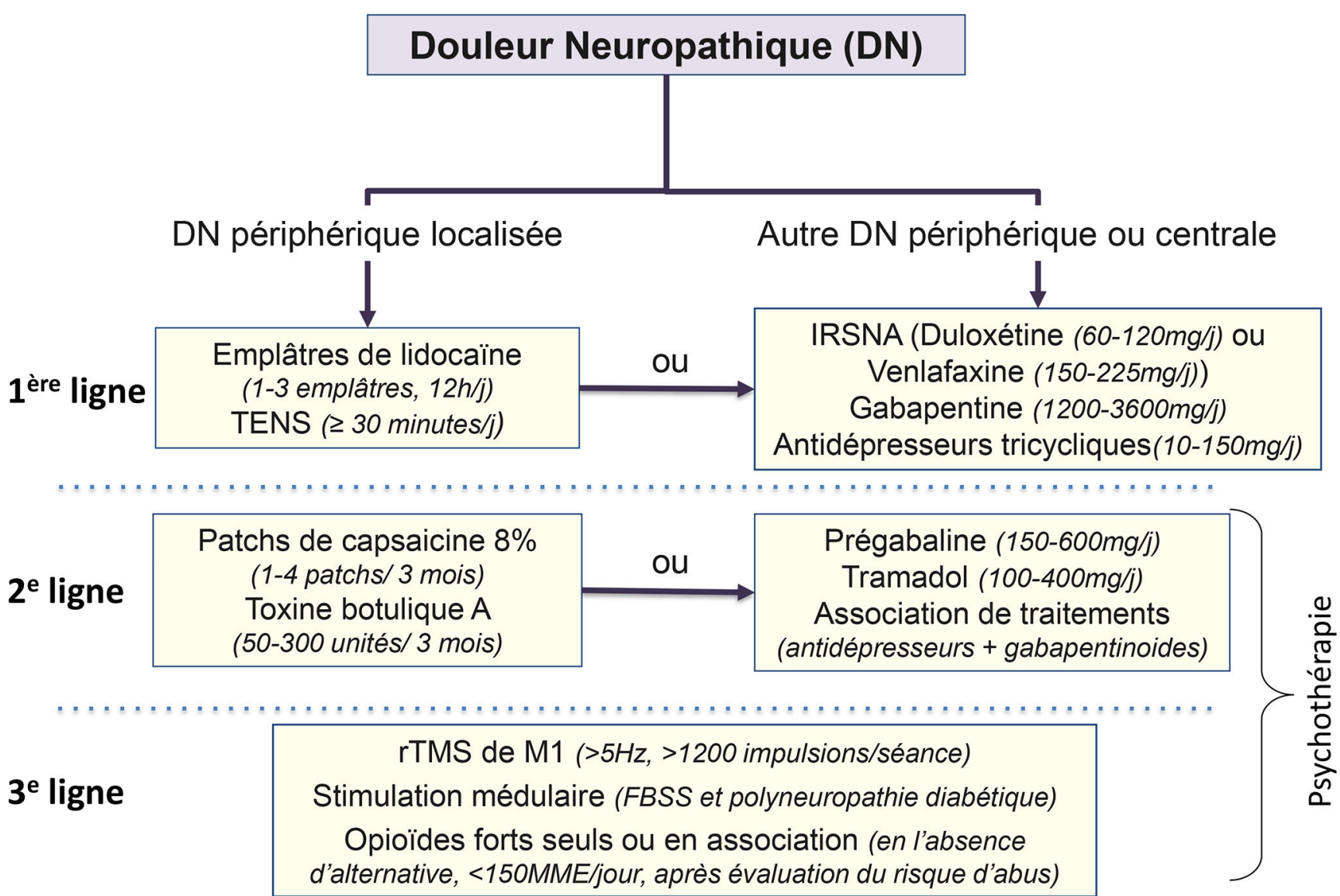

Fig. 1 Algorithme thérapeutique proposé pour la prise en charge de la douleur neuropathique de l'adulte. TENS : transcutaneous electrical nerve stimulation ; IRSNA : antidépresseur inhibiteur de recapture de la sérotonine et de la noradrénaline ; rTMS : repetitive transcranial magnetic stimulation

son efficacité par voie sous-cutanée dans les douleurs neuropathiques périphériques d'étendue limitée [28-30], mais ne dispose pas d'AMM dans cette indication. Ces deux traitements ont un ratio efficacité/effets indésirables plutôt meilleur que celui des traitements systémiques et ont tous deux une efficacité rémanente d'environ trois mois après leur utilisation [28,30,31]. Cependant, contrairement aux patchs de lidocaïne, nous proposons ces deux traitements en deuxième intention (pour les DN périphériques avec zone douloureuse peu étendue), en raison de leur coût (235 euros/patch de capsaïcine en France en 2019 ; environ 200 euros pour un flacon de 100 unités de toxine botulique A) et surtout de leur usage strictement hospitalier rendant difficile leur prescription en médecine de ville en France.

Pour l'élaboration de ces recommandations, les AMM et les conditions d'accès n'ont pas été pris en compte, ces paramètres pouvant être différents selon les pays et évoluer dans le temps pour un pays donné. Certaines AMM françaises en 2020 sont néanmoins spécifiées dans la discussion. Il est important de rappeler que certains éléments peuvent jus- tifier une telle prescription [32] : d'une part, l'existence de données scientifiques suffisantes (telles que des recommandations) et, d'autre part, la notion de " meilleur soin sans augmentation de risque ». En effet, le strict respect de l'AMM ne coïncide pas toujours avec la meilleure prise en charge thérapeutique du patient, c'est-à-dire avec l'obligation déontologique et légale de lui procurer les meilleurs soins. La notion de meilleur soin est bien applicable au contexte de la douleur neuropathique, chronique, réfractaire et invalidante. La situation est plus ambiguë pour les traitements de première ligne, où l'on ne peut pas, par définition, évoquer de caractère réfractaire.

Une dernière évolution notable de nos recommandations par rapport à celles de 2010 concerne les opioïdes. Bien que la place des opioïdes dans notre algorithme thérapeutique soit inchangée (le tramadol restant recommandé en deuxième intention avec 7/7 études positives et les opioïdes forts en troisième intention), les préconisations concernant la surveillance du risque de mésusage aux opioïdes sont beaucoup plus strictes. Cela tient essentiellement à la « crise des 
opioïdes » qui est devenue un véritable fléau de santé publique depuis 2010, notamment aux États-Unis. En effet, les opioïdes prescrits ont causé la mort de milliers de personnes aux États-Unis (plus de 17000 par an actuellement selon les derniers chiffres disponibles [drugabuse.gov]). Bien que la situation ne soit pas aussi dramatique en France, les prescriptions de certains opioïdes tels que l'oxycodone ont nettement augmenté [33]. Il est donc essentiel de respecter les recommandations actuelles de prescription en évaluant le risque d'abus ou de mésusage pour tous les opioïdes, incluant le tramadol [34-36]. Pour la prise en charge de la DN, les opioïdes forts ne doivent donc être utilisés qu'en dernier recours, en l'absence d'alternative, et pour la durée la plus courte possible.

Les techniques de neurostimulation invasives ou non invasives apparaissent pour la première fois au même titre que les traitements pharmacologiques dans un algorithme thérapeutique pour les DN. La stimulation électrique transcutanée (TENS) est recommandée en première intention pour les DN périphériques localisées. Cependant, l'accès à ce traitement reste difficile en France, celui-ci ne pouvant être prescrit que par une structure spécialisée douleur chronique (SDC). Compte tenu de la facilité d'utilisation et de la sécurité d'emploi de cette technique de stimulation, nous estimons que ce traitement pourrait bénéficier à beaucoup plus de patients présentant une $\mathrm{DN}$ et devrait pouvoir à terme être proposé en médecine de ville. La stimulation magnétique transcrânienne répétitive à haute fréquence du cortex moteur (rTMS), technique non invasive et généralement bien tolérée de neurostimulation consistant à appliquer un champ magnétique sur le cortex cérébral de façon indolore au moyen d'une bobine, et la stimulation médullaire implantable sont désormais recommandées en troisième intention. La rTMS peut être proposée pour toute douleur neuropathique, alors que les indications de la stimulation médullaire sont limitées aux radiculalgies chroniques postchirurgicales et à la polyneuropathie diabétique douloureuse, et ce, dès un an d'évolution de la douleur. Même si le nombre de centres spécialisés proposant ces techniques reste limité en France à l'heure actuelle, la stimulation médullaire en particulier est très largement utilisée dans d'autres pays européens comme la Belgique ou les Pays-Bas. Il nous semble important que les praticiens aient une meilleure connaissance de ces techniques et de leur efficacité pour adresser leurs patients douloureux neuropathiques vers des centres spécialisés.

Parmi les approches validées pour la prise en charge des douleurs neuropathiques figurent enfin les psychothérapies, dont la TCC et la thérapie de pleine conscience. Ces techniques ont l'avantage d'une innocuité quasi absolue lorsqu'elles sont effectuées selon les recommandations, c'est-à-dire précédées par une évaluation psychologique et psychopathologique permettant de repérer des antécédents psychiatriques et d'adapter la prise en charge en fonction du patient. Il convient notamment d'être vigilant en cas d'antécédents de psychotraumatismes. Nous recommandons les psychothérapies en deuxième intention, en association avec les autres traitements.

Nous n'avons retrouvé aucune étude de qualité élevée portant spécifiquement sur les enfants et les personnes âgées. Chez l'enfant, nous recommandons en première intention la gabapentine (10 à $30 \mathrm{mg} / \mathrm{kg}$ par jour en trois prises) ou l'amitriptyline $(0,3$ à $1 \mathrm{mg} / \mathrm{kg}$ par jour en une prise vespérale), ces derniers pouvant être associés en cas de soulagement insuffisant. Les autres traitements validés chez l'adulte peuvent être proposés, en particulier les emplâtres de lidocaïne, en cas de douleur neuropathique périphérique localisée. Un soutien psychologique et une prise en charge en kinésithérapie sont également recommandés pour améliorer la fonction et minimiser l'impact sur le développement de l'enfant. En cas d'échec de ces traitements, il est souhaitable de s'orienter vers une structure douleur pédiatrique. Chez le sujet âgé, en l'absence d'études spécifiques, nous proposons les gabapentinoïdes, les emplâtres de lidocaïne et la duloxétine en première intention, suivant ainsi les dernières propositions d'un consensus d'experts en gériatrie [37], alors que les antidépresseurs tricycliques sont à envisager seulement en deuxième intention du fait de leurs effets indésirables cardiaques et anticholinergiques plus fréquents chez les sujets âgés. Dans tous les cas, une titration lente est recommandée pour les molécules par voie systémique, afin d'optimiser la tolérance.

\section{Conclusion}

Nous proposons pour la première fois un algorithme thérapeutique prenant en compte l'ensemble des approches thérapeutiques validées des douleurs neuropathiques. Il nous semble essentiel que ces nouvelles recommandations soient diffusées le plus largement possible au sein de la communauté des professions médicales francophones. Jusqu'à présent, l'évaluation des pratiques avait révélé une connaissance limitée des traitements de première intention de ces douleurs $[7,9]$. Nous espérons que ces nouvelles recommandations contribueront à une formation continue de qualité pour optimiser la prise en charge des patients douloureux.

Remerciements Nous souhaitons remercier tous les membres du groupe de lecture : Nicolas Authier, Christine Berlemont, Célian Bertin, Eric Bozzolo, Bruno Brochet, Martine Chauvin, Elisabeth Collin, Virginie Corand-Dousset, Christelle Créac'h, Thierry Delorme, Marie-Christine Djian, Anne Donnet, Bénédicte Eschalier, Denys Fontaine, Pierric Giraud, Aline Le Chevalier, Christine Leveque, Gaelle Martine, Valeria Martinez, Anne Masselin-Dubois, Kamel 
Mechtouf, Patrick Mertens, Julien Nizard, Michel Olivier, Serge Perrot, Roland Peyron, Daniele Ranoux, Catherine Sebire, Denis Sinardet, Gregory Tosti, Pascale VergneSalle, Eric Viel, Wood Chantal

Liens d'intérêts : $X$. Moisset a reçu des honoraires de Lilly, TBWA, Teva, Novartis, Roche, Biogen, Sanofi-Genzyme et Merck-Serono, et un soutien non financier SOS oxygène, Boehringer, Bristol-Myers-Squibb, sans lien avec ce travail.

H. Alchaar a reçu des honoraires de Novartis, Sanofi, MSD, sans lien avec ce travail.

\section{J. Avez Couturier déclare ne pas avoir de lien d'intérêt.}

D. Bouhassira a reçu des honoraires de Grünenthal, Novartis and Air liquide, sans lien avec ce travail et participe aux projets européens DOLORISK et IMI PainCare.

S. Conradi déclare ne pas avoir de lien d'intérêt.

M.-H. Delmotte a reçu des honoraires de Mylan, sans lien avec ce travail.

M. Lantéri-Minet a reçu des financements directs ou indirects d'Allergan, Amgen, Astellas, ATI, BMS, Boehringer, Boston Scientific, CoLucid, Convergence, GlaxoSmithKline, Grünenthal, Eli Lilly, Medtronic, Menarini, MSD, Novartis, Pfizer, Reckitt Benckiser, Saint-Jude, SanofiAventis, Teva Pharmaceuticals, UCB et Zambon.

J.-P. Lefaucheur a reçu des honoraires de Novo Nordisk, Pfizer, et Sanofi sans lien avec ce travail.

G. Mick a reçu des honoraires de Sanofi-MSD, Lilly, Pfizer, Teva, Grünenthal, Mylan, BMS UPSA sans lien avec ce travail.

V. Piano a reçu des honoraires de Novartis, Grünenthal, Ethipharm, Mylan, sans lien avec ce travail.

G. Pickering a reçu des honoraires de Sanofi, MSD, Grünenthal, Mylan, Kyowa Kirin sans lien avec ce travail.

E. Piquet a reçu des honoraires de Grünenthal et Medtronic, sans lien avec ce travail.

C. Regis déclare ne pas avoir de lien d'intérêt.

E. Salvat a reçu des honoraires de Novartis, Sanofi, Ethypharm, Grünenthal, CSL Behring, MSD, Merz, Kyowa Kirin, Mundipharma, Mylan, Genzyme, Medtronic, Abbott et Boston sans lien avec ce travail.

N. Attal a reçu des honoraires de Sanofi-MSD, SanofiAventis, Sanofi-Pasteur-MSD, MSD vaccins, Ethypharm, Lilly France, Grünenthal, Ipsen, Astellas, sans lien avec ce travail et participe au consortium européen DOLORISK.

\section{Références}

1. Doth AH, Hansson PT, Jensen MP, Taylor RS (2010) The burden of neuropathic pain: a systematic review and meta-analysis of health utilities. Pain 149:338-44. https://doi.org/10.1016/j. pain.2010.02.034

2. Attal N, Lantéri-Minet M, Laurent B, et al (2011) The specific disease burden of neuropathic pain: results of a French nationwide survey. Pain 152:2836-43. https://doi.org/10.1016/j. pain.2011.09.014

3. Langley PC, Van Litsenburg C, Cappelleri JC, Carroll D (2013) The burden associated with neuropathic pain in Western Europe. J Med Econ 16:85-95. https://doi.org/10.3111/13696998. 2012.729548

4. Colloca L, Ludman T, Bouhassira D, et al (2017) Neuropathic pain. Nat Rev Dis Primer 3:17002. https://doi.org/10.1038/ nrdp. 2017.2

5. Dworkin RH, Malone DC, Panarites CJ, et al (2010) Impact of postherpetic neuralgia and painful diabetic peripheral neuropathy on health care costs. J Pain 11:360-8. https://doi.org/10.1016/j. jpain.2009.08.005

6. Bouhassira D, Letanoux M, Hartemann A (2013) Chronic pain with neuropathic characteristics in diabetic patients: a French cross-sectional study. PloS One 8:e74195. https://doi.org/ 10.1371/journal.pone.0074195

7. Piano V, Lantéri-Minet M, Steegers M, et al (2013) A case vignette study to assess the knowledge of pain physicians of neuropathic cancer pain: room for improvement. Pain Physician 16: E779-E88

8. Torrance N, Ferguson JA, Afolabi E, et al (2013) Neuropathic pain in the community: more under-treated than refractory? Pain 154:690-9. https://doi.org/10.1016/j.pain.2012.12.022

9. Martinez V, Attal N, Vanzo B, et al (2014) Adherence of French GPs to chronic neuropathic pain clinical guidelines: results of a cross-sectional, randomized, "e" case-vignette survey. PloS One 9:e93855. https://doi.org/10.1371/journal.pone.0093855

10. Heutink M, Post MWM, Wollaars MM, van Asbeck FWA (2011) Chronic spinal cord injury pain: pharmacological and nonpharmacological treatments and treatment effectiveness. Disabil Rehabil 33:433-40. https://doi.org/10.3109/09638288.2010. 498557

11. Tamburin S, Lacerenza MR, Castelnuovo G, et al (2016) Pharmacological and non-pharmacological strategies in the integrated treatment of pain in neurorehabilitation. Evidence and recommendations from the Italian Consensus Conference on Pain in Neurorehabilitation. Eur J Phys Rehabil Med 52:741-52

12. Martinez V, Attal N, Bouhassira D, Lantéri-Minet M (2010) Les douleurs neuropathiques chroniques : diagnostic, évaluation et traitement en médecine ambulatoire. Recommandations pour la pratique clinique de la Société française d'étude et de traitement de la douleur. Douleurs : Évaluation - Diagnostic - Traitement 11:3-21. https://doi.org/10.1016/j.douler.2009.12.009

13. Finnerup NB, Attal N, Haroutounian S, et al (2015) Pharmacotherapy for neuropathic pain in adults: a systematic review and meta-analysis. Lancet Neurol 14:162-73. https://doi.org/ 10.1016/S1474-4422(14)70251-0

14. Dworkin RH, O'Connor AB, Kent J, et al (2013) Interventional management of neuropathic pain: NeuPSIG recommendations. Pain 154:2249-61. https://doi.org/10.1016/j.pain.2013.06.004

15. Cruccu G, Garcia-Larrea L, Hansson P, et al (2016) EAN guidelines on central neurostimulation therapy in chronic pain conditions. Eur J Neurol 23:1489-99. https://doi.org/10.1111/ ene. 13103

16. AFFSAPS (2009) Prise en charge médicamenteuse de la douleur aiguë et chronique chez l'enfant 
17. Moisset X, Bouhassira D, Avez Couturier J, et al (2020) Pharmacological and non-pharmacological treatments for neuropathic pain: systematic review and French recommendations. Rev Neurol (Paris) 176:325-52 https://doi.org/10.1016/j.neurol.2020. 01.361

18. Guyatt GH, Oxman AD, Vist GE, et al (2008) GRADE: an emerging consensus on rating quality of evidence and strength of recommendations. BMJ 336:924-6. https://doi.org/10.1136/ bmj.39489.470347.AD

19. Balshem H, Helfand M, Schünemann HJ, et al (2011) GRADE guidelines: 3. Rating the quality of evidence. J Clin Epidemiol 64:401-6. https://doi.org/10.1016/j.jclinepi.2010.07.015

20. Bendtsen L, Zakrzewska JM, Abbott J, et al (2019) EAN guideline on trigeminal neuralgia. Eur J Neurol 26(6) :831-49 https:// doi.org/10.1111/ene. 13950

21. Heutink M, Post MW, Luthart P, et al (2014) Long-term outcomes of a multidisciplinary cognitive behavioural programme for coping with chronic neuropathic spinal cord injury pain. J Rehabil Med 46:540-5. https://doi.org/10.2340/16501977-1798

22. Brown S, Johnston B, Amaria K, et al (2016) A randomized controlled trial of amitriptyline versus gabapentin for complex regional pain syndrome type I and neuropathic pain in children. Scand J Pain 13:156-63. https://doi.org/10.1016/j.sjpain. 2016.05.039

23. Pickering G, Voute M, Macian N, et al (2019) Effectiveness and safety of 5\% lidocaine-medicated plaster on localized neuropathic pain after knee surgery: a randomized, double-blind controlled trial. Pain 160:1186-95. https://doi.org/10.1097/j. pain.0000000000001502

24. Demant DT, Lund K, Finnerup NB, et al (2015) Pain relief with lidocaine $5 \%$ patch in localized peripheral neuropathic pain in relation to pain phenotype: a randomized, double-blind, and placebo-controlled, phenotype panel study. Pain 156:2234-44. https://doi.org/10.1097/j.pain.0000000000000266

25. Finnerup NB, Haroutounian S, Baron R, et al (2018) Neuropathic pain clinical trials: factors associated with decreases in estimated drug efficacy. Pain 159:2339-46. https://doi.org/10.1097/j. pain. 0000000000001340

26. Robertson K, Marshman LAG, Plummer D, Downs E (2019) Effect of gabapentin vs. pregabalin on pain intensity in adults with chronic sciatica: a randomized clinical trial. JAMA Neurol 76:28-34. https://doi.org/10.1001/jamaneurol.2018.3077

27. Driot D, Jouanjus E, Oustric S, et al (2019) Patterns of gabapentin and pregabalin use and misuse: results of a population-based cohort study in France. Br J Clin Pharmacol 85:1260-9. https:// doi.org/10.1111/bcp. 13892

28. Attal N, de Andrade DC, Adam F, et al (2016) Safety and efficacy of repeated injections of botulinum toxin A in peripheral neuropathic pain (BOTNEP): a randomised, double-blind, placebo-controlled trial. Lancet Neurol 15:555-65. https://doi. org/10.1016/S1474-4422(16)00017-X

29. Ghasemi M, Ansari M, Basiri K, Shaigannejad V (2014) The effects of intradermal botulinum toxin type a injections on pain symptoms of patients with diabetic neuropathy. J Res Med Sci 19:106-11

30. Han ZA, Song DH, Oh HM, Chung ME (2016) Botulinum toxin type A for neuropathic pain in patients with spinal cord injury. Ann Neurol 79:569-78. https://doi.org/10.1002/ana.24605

31. Derry S, Rice AS, Cole P, et al (2017) Topical capsaicin (high concentration) for chronic neuropathic pain in adults. Cochrane Database Syst Rev 1:CD007393. https://doi.org/10.1002/ 14651858.CD007393.pub4

32. Bouvenot G, Juillet Y, Saint-Pierre A, Serre MP (2018) Les prescriptions médicamenteuses hors AMM (autorisation de mise sur le marché) en France. Une clarification est indispensable. Bull Acad Natl Med 202:1749-82. https://doi.org/10.1016/S00014079(19)30181-5

33. Chenaf C, Kaboré JL, Delorme J, et al (2019) Prescription opioid analgesic use in France: Trends and impact on morbidity-mortality. Eur J Pain Lond Engl 23:124-34. https://doi.org/10.1002/ ejp. 1291

34. Moisset X, Trouvin AP, Tran VT, et al (2016) Use of strong opioids in chronic non-cancer pain in adults. Evidence-based recommendations from the French Society for the Study and Treatment of Pain. Presse Med Paris Fr 45:447-62. https://doi. org/10.1016/j.lpm.2016.02.014

35. Miotto K, Cho AK, Khalil MA, et al (2017) Trends in tramadol: pharmacology, metabolism, and misuse. Anesth Analg 124:44 51. https://doi.org/10.1213/ANE.0000000000001683

36. ANSM (2019) État des lieux de la consommation des antalgiques opioïdes et leurs usages problématiques. https://ansm.sante.fr/Sinformer/Points-d-information-Points-d-information/Antalgiquesopioides-1-ANSM-publie-un-etat-des-lieux-de-la-consommationen-France-Point-d-Information. Accessed 19 Jul 2019

37. Pickering G, Marcoux M, Chapiro S, et al (2016) An algorithm for neuropathic pain management in older people. Drugs Aging 33:575-83. https://doi.org/10.1007/s40266-016-0389-7 